\title{
Top-assisted di-Higgs boson production motivated by baryogenesis
}

\author{
Wei-Shu Hou, Masaya Kohda, and Tanmoy Modak \\ Department of Physics, National Taiwan University, Taipei 10617, Taiwan
}

(Received 14 January 2019; published 29 March 2019)

\begin{abstract}
We study top-assisted di-Higgs production via $c g \rightarrow t H \rightarrow t h h$, where $h$ is the $125 \mathrm{GeV}$ scalar boson and $H$ is the $C P$-even heavy Higgs. The context is the two Higgs doublet model without a $Z_{2}$ symmetry, where the extra Yukawa coupling $\rho_{t c}$ generates $t H$ production, with the extra top Yukawa $\rho_{t t} \simeq 0$ to avoid $g g \rightarrow H$ constraints. We find that discovery is possible for $m_{H}$ around $300 \mathrm{GeV}$ or so at the LHC but would need finite $h-H$ mixing angle $\cos \gamma$ to allow for finite $\lambda_{H h h}$ coupling, and $\rho_{t c}$ also needs to be not too small. A sizable $\rho_{t c}$ could drive electroweak baryogenesis, which further motivates the search.
\end{abstract}

DOI: 10.1103/PhysRevD.99.055046

\section{INTRODUCTION}

The highlight at the LHC so far is the discovery of the $125 \mathrm{GeV}$ scalar boson $h$ [1] in 2012, which resembles rather closely [2] the Higgs boson of the Standard Model (SM). To improve our understanding of the Higgs potential, a key goal at the LHC is to search for di-Higgs, or $p p \rightarrow h h$, production. The program is rather challenging, as $h h$ production in the SM $[3,4]$ is rather suppressed. The ATLAS [5-7] and CMS [8-11] experiments have already conducted searches for resonant and nonresonant di-Higgs production, but there is little expectation that the SM process can be observed even at the High-Luminosity LHC (HL-LHC). In this paper, we explore a novel possibility with new physics, that of resonant $h h$ production in association with a top quark.

The context is a two Higgs doublet model (2HDM) with extra Yukawa couplings, i.e., without a discrete $Z_{2}$ symmetry to impose the Glashow-Weinberg [12] natural flavor conservation (NFC) condition to forbid flavor-changing neutral Higgs $(\mathrm{FCNH})$ couplings. Note that the usual $Z_{2}$ symmetry eliminates all extra Yukawa couplings. Two processes that feed di-Higgs production can be operative. The existence of extra diagonal Yukawa coupling $\rho_{t t}$ of the exotic $C P$-even neutral Higgs boson $H$ means that one could have $g g \rightarrow H \rightarrow h h$ production through a triangle top-quark loop. A second process depends on the FCNH $t c H$ coupling, $\rho_{t c}$, whereby one can have $c g \rightarrow t H \rightarrow t h h$ (conjugate process implied). In a previous paper [13], we considered turning $\rho_{t t}$ off, so the first process is subdued, and one is left with the second process: di-Higgs and

Published by the American Physical Society under the terms of the Creative Commons Attribution 4.0 International license. Further distribution of this work must maintain attribution to the author(s) and the published article's title, journal citation, and DOI. Funded by SCOAP . top-associated production. In this paper, we focus on this process, exploiting the extra top quark to investigate possible prospects at the LHC.

We stress that $2 \mathrm{HDM}$ without extra $Z_{2}$ symmetry could [14] account for baryon asymmetry of the Universe, via electroweak baryogenesis (EWBG). The leading mechanism is via $\rho_{t t}$ and is rather robust. However, in exploring [13] the prospect for a lighter pseudoscalar $A$ boson around $300 \mathrm{GeV}$, in the face of direct search bounds, we opted to turn off $\rho_{t t}$, noting that $\rho_{t c} \sim 1$ would still offer an alternative mechanism [14] for EWBG, and hence it is interesting in itself. Thus, the $\rho_{t c}$ driven $c g \rightarrow t H \rightarrow t h h$ process studied here is a companion to the $c g \rightarrow t A \rightarrow t t \bar{c}$ process that bears a rather intriguing signature.

We find that $t h h$ discovery is possible at the HL-LHC for relatively light $H$, where the associated top quark gives extra handle on background reduction. However, a relatively large $H h h$ coupling would be needed; hence, the prospect cannot be said to be very likely, but it is not negligible. In the following, we start with the formalism in Sec. II, then continue with the collider signatures in Sec. III, and end with some discussions in Sec. IV.

\section{FORMALISM}

The $C P$-even scalars $h$ and $H$ and the $C P$-odd scalar $A$ couple to fermions by $[15,16]$

$$
\begin{aligned}
- & \frac{1}{\sqrt{2}} \sum_{F=U, D, L} \bar{F}_{i L}\left[\left(-\lambda_{i j}^{F} s_{\gamma}+\rho_{i j}^{F} c_{\gamma}\right) h\right. \\
& \left.+\left(\lambda_{i j}^{F} c_{\gamma}+\rho_{i j}^{F} s_{\gamma}\right) H-i \operatorname{sgn}\left(Q_{F}\right) \rho_{i j}^{F} A\right] F_{j R}+\text { H.c. }
\end{aligned}
$$

where $i, j=1,2,3$ are generation indices that are summed over and $\lambda_{i j}^{F}=\left(\sqrt{2} m_{i}^{F} / v\right) \delta_{i j}$ (with $v \simeq 246 \mathrm{GeV}$ ) and $\rho^{F}$ are $3 \times 3$ real diagonal and complex matrices, respectively. With shorthand $c_{\gamma}=\cos \gamma, s_{\gamma}=\sin \gamma$, the mixing angle $\gamma$ is 
usually written as $\alpha-\beta$ in Type-II $2 \mathrm{HDM}$ notation. However, as we advocate no $Z_{2}$ symmetry and there exists a second set of Yukawa couplings $\rho_{i j}^{F}$, we prefer the notation of Ref. [17], since $\tan \beta$ is ill defined. The FCNH couplings of interest for $t c H$ are $\rho_{23}^{U} \equiv \rho_{c t}$ and $\rho_{32}^{U} \equiv \rho_{t c}$. $B$ physics sets stringent limits on $\rho_{c t}$ [16], while $\rho_{t c}$ is only mildly constrained [18], depending on $m_{H^{+}}$. In our study, we set $\rho_{c t}=0$ and take $\left|\rho_{t c}\right|<1$.

The most general $C P$-conserving two Higgs doublet potential is given in the Higgs basis as $[15,17]$

$$
\begin{aligned}
V\left(\Phi, \Phi^{\prime}\right)= & \mu_{11}^{2}|\Phi|^{2}+\mu_{22}^{2}\left|\Phi^{\prime}\right|^{2}-\left(\mu_{12}^{2} \Phi^{\dagger} \Phi^{\prime}+\text { H.c. }\right) \\
& +\frac{\eta_{1}}{2}|\Phi|^{4}+\frac{\eta_{2}}{2}\left|\Phi^{\prime}\right|^{4}+\eta_{3}|\Phi|^{2}\left|\Phi^{\prime}\right|^{2}+\eta_{4}\left|\Phi^{\dagger} \Phi^{\prime}\right|^{2} \\
& +\left[\frac{\eta_{5}}{2}\left(\Phi^{\dagger} \Phi^{\prime}\right)^{2}+\left(\eta_{6}|\Phi|^{2}+\eta_{7}\left|\Phi^{\prime}\right|^{2}\right) \Phi^{\dagger} \Phi^{\prime}+\text { H.c. }\right],
\end{aligned}
$$

where $v$ arises from the doublet $\Phi$ via $\mu_{11}^{2}=-\frac{1}{2} \eta_{1} v^{2}$, while $\left\langle\Phi^{\prime}\right\rangle=0$ (hence, $\mu_{22}^{2}>0$ ), and $\eta_{i}$ s are quartic couplings, again in the notation of Ref. [17]. A second minimization condition, $\mu_{12}^{2}=\frac{1}{2} \eta_{6} v^{2}$, removes $\mu_{12}^{2}$ and reduces the total number of parameters to nine [17]. The mixing angle $\gamma$ between the $C P$-even bosons satisfies the relations [17]

$$
c_{\gamma}^{2}=\frac{\eta_{1} v^{2}-m_{h}^{2}}{m_{H}^{2}-m_{h}^{2}}, \quad \sin 2 \gamma=\frac{2 \eta_{6} v^{2}}{m_{H}^{2}-m_{h}^{2}},
$$

for which, for $c_{\gamma}$ small but not infinitesimal, one has $c_{\gamma} \simeq\left|\eta_{6}\right| v^{2} /\left(m_{H}^{2}-m_{h}^{2}\right)$. This is approximate alignment [17]; i.e., small $c_{\gamma}$ values can be attained without requiring $\eta_{6}$ to be small. But in the alignment limit, $c_{\gamma} \rightarrow 0$, either [17] $\eta_{6}$ has to vanish (and $m_{h}^{2} \rightarrow \eta_{1} v^{2}$ ) or else one has decoupling [19], i.e., $m_{H}^{2} / v^{2} \gg 1$.

We are interested in the Hhh coupling, which is the coefficient of the $\lambda_{H h h} H h^{2}$ term derivable from Eq. (2),

$$
\begin{aligned}
\lambda_{H h h}= & \frac{v}{2}\left[3 c_{\gamma} s_{\gamma}^{2} \eta_{1}+c_{\gamma}\left(3 c_{\gamma}^{2}-2\right) \eta_{345}\right. \\
& \left.+3 s_{\gamma}\left(1-3 c_{\gamma}^{2}\right) \eta_{6}+3 s_{\gamma} c_{\gamma}^{2} \eta_{7}\right],
\end{aligned}
$$

with $\eta_{345}=\eta_{3}+\eta_{4}+\eta_{5}$. It reduces further to

$\lambda_{H h h} \simeq \frac{c_{\gamma}}{2} v\left[3 \frac{m_{H}^{2}}{v^{2}}-2 \eta_{345}+3 \operatorname{sgn}\left(s_{\gamma}\right) c_{\gamma} \eta_{7}+\mathcal{O}\left(c_{\gamma}^{2}\right)\right]$,

for small $c_{\gamma}$, so $\lambda_{H h h} \rightarrow 0$ as $c_{\gamma} \rightarrow 0$. To enhance $c g \rightarrow t H \rightarrow t h h$, sizable $\lambda_{H h h}$ is needed, and $\eta_{345}<0$ may be preferred so the first two terms add up. However, $\lambda_{H h h}$ could still be sizable if $2 \eta_{345} \gg 3 m_{H}^{2} / v^{2}>0$. Either way, a large $\left|\eta_{7}\right|$ with a proper sign for $\eta_{7} c_{\gamma} s_{\gamma}$ would help.
The quartic couplings $\eta_{1}, \eta_{3-6}$ can be expressed in terms of $m_{h}, m_{A}, m_{H}, m_{H^{ \pm}}$, and $\mu_{22}$, all normalized to $v$, as well as the mixing angle $\gamma$ [17],

$$
\begin{aligned}
& \eta_{1}=\frac{m_{h}^{2} s_{\gamma}^{2}+m_{H}^{2} c_{\gamma}^{2}}{v^{2}}, \\
& \eta_{3}=\frac{2\left(m_{H^{ \pm}}^{2}-\mu_{22}^{2}\right)}{v^{2}}, \\
& \eta_{4}=\frac{m_{h}^{2} c_{\gamma}^{2}+m_{H}^{2} s_{\gamma}^{2}-2 m_{H^{ \pm}}^{2}+m_{A}^{2}}{v^{2}}, \\
& \eta_{5}=\frac{m_{H}^{2} s_{\gamma}^{2}+m_{h}^{2} c_{\gamma}^{2}-m_{A}^{2}}{v^{2}}, \\
& \eta_{6}=\frac{\left(m_{h}^{2}-m_{H}^{2}\right)\left(-s_{\gamma}\right) c_{\gamma}}{v^{2}},
\end{aligned}
$$

but $\eta_{2}$ and $\eta_{7}$ are not related to masses, nor the mixing angle $\gamma$. Thus, we take $v, \gamma, m_{h}, m_{A}, m_{H}, m_{H^{ \pm}}, \mu_{22}, \eta_{2}$, and $\eta_{7}$ as the phenomenological parameters. To save computation time, we randomly generate these parameters in the following ranges: $\mu_{22} \in[0,700] \mathrm{GeV}, m_{H} \in[250,500] \mathrm{GeV}$, $m_{H^{ \pm}} \in[300,600] \mathrm{GeV}, \eta_{2} \in[0,3], \eta_{7} \in[-3,3]$, and $\gamma$ values that satisfy $c_{\gamma} \in[0,0.2]$, with $m_{h}=125 \mathrm{GeV}$. We choose two different scenarios for $m_{A}$. In the main scenario, we generate $m_{A} \in[250,500] \mathrm{GeV}$, with $m_{H}<m_{A}, m_{H^{ \pm}}$. In the second scenario, we take $\left|m_{A}-m_{H}\right|<\delta$, where the choice of $\delta$ is discussed later in the section. We explore up to $m_{H}=500 \mathrm{GeV}$ because, while $\lambda_{H h h}$ and $\mathcal{B}(H \rightarrow h h)$ increases with $m_{H}$, the discovery potential for $c g \rightarrow t H \rightarrow$ thh suffers the drop in parton luminosities for heavier $m_{H}$.

The dynamical parameters in the Higgs potential, Eq. (2), need to satisfy perturbativity, tree-level unitarity, and positivity conditions, for which we utilize $2 \mathrm{HDMC}$ [20]. 2HDMC uses the input parameters [20] $m_{H^{ \pm}}$and $\Lambda_{1-7}$ in the Higgs basis and with $v$ implicit. We identify $\Lambda_{1-7}$ with $\eta_{1-7}$. Further, we conservatively demand all $\left|\eta_{i}\right| \leq 3$, while $\eta_{2}>0$ is required by the potential positivity, in addition to more involved conditions for other couplings. To match the convention of $2 \mathrm{HDMC}$, we take $-\pi / 2 \leq \gamma \leq \pi / 2$.

We also need to impose the stringent oblique $T$ parameter [21] constraint, which constrains the scalar masses $m_{H}$, $m_{A}$, and $m_{H^{ \pm}}[22,23]$, and hence $\eta_{i}$ s. We apply the $T$ parameter constraint [24] on the points that passed $2 \mathrm{HDMC}$, using the expression given in Ref. [23]. The final "scanned points" within $2 \sigma$ error of the $T$ parameter are plotted in Fig. 1. The upper panel is for $m_{H}<m_{H^{ \pm}}, m_{A}$, such that $H \rightarrow A Z, H^{ \pm} W^{\mp}$ decays are disallowed, which in turn enhances the $H \rightarrow h h$ branching ratio. As expected, the upper range for $\lambda_{H h h}$ mildly increases as $m_{H}$ becomes heavier but vanishes with $c_{\gamma} \rightarrow 0$. 

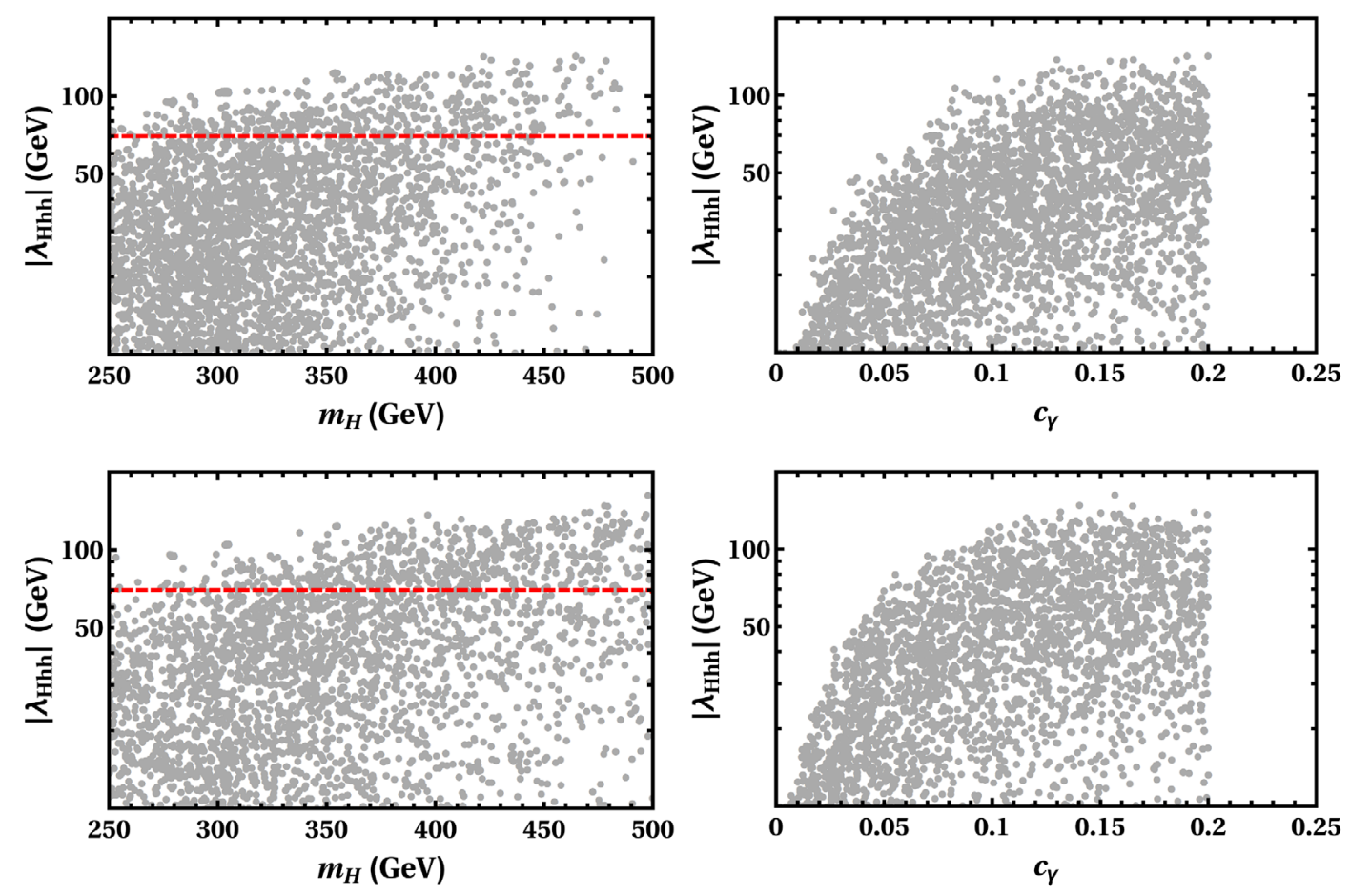

FIG. 1. The $\lambda_{H h h}$ vs $m_{H}$ and $c_{\gamma}$ plots for the scan points that pass perturbativity, tree-level unitarity, and positivity through 2HDMC, where $\left|\eta_{i}\right|<3$ is maintained. The $T$ parameter constraint is also imposed. Upper panels are for $m_{H}<m_{A}, m_{H^{ \pm}}$, and lower panels are for $\left|m_{A}-m_{H}\right|<5 \mathrm{GeV}$. See the text for detailed explanation.

The FCNH coupling $\rho_{t c}$ also receives constraint from CMS four-top search [25] through the $c g \rightarrow t H \rightarrow t t \bar{c}$ process, which is proportional to $\left|s_{\gamma} \rho_{t c}\right|^{2}$ [13] if we take $\rho_{c t}=0$. However, if $A$ and $H$ are mass and width degenerate, the processes $c g \rightarrow t A \rightarrow t t \bar{c}$ and $c g \rightarrow t H \rightarrow$ $t t \bar{c}$ cancel each other exactly [13,26], resulting in a potentially much weaker constraint on $\rho_{t c}$, which can in principle give rise to larger thh production. The lower panels of Fig. 1 are for this scenario of nearly degenerate $A$ and $H$, where we assume $\left|m_{A}-m_{H}\right|<\delta$, with $\delta=5 \mathrm{GeV}$ for illustration. The dependence of $\lambda_{H h h}$ on $m_{H}$ and $c_{\gamma}$ is similar to that in the $m_{H}<m_{H^{ \pm}}, m_{A}$ case. We note that in the left panels, i.e., $\lambda_{H h h}$ vs $m_{H}$, we have drawn a line at $\lambda_{H h h} \simeq 70 \mathrm{GeV}$ to illustrate that $\lambda_{H h h}$ can be sizable over a finite parameter region.

\section{COLLIDER SIGNATURE}

The discovery potential of the $c g \rightarrow t H \rightarrow t h h$ (conjugate process implied) depends on the $h h$ decay final states. In this paper, we primarily focus on $t \rightarrow b \ell^{+} \nu_{\ell}(\ell=e, \mu)$ with both $h$ bosons decaying via $h \rightarrow b \bar{b}$, giving rise to five $b$ jets, one lepton, and the missing transverse energy $\left(E_{T}^{\text {miss }}\right)$ signature. We do not look for hadronic decay of $t$ due to QCD multijet backgrounds, as also discussed in Ref. [27]. In general, $h h \rightarrow \gamma \gamma b \bar{b}$ and $h h \rightarrow \tau \bar{\tau} b \bar{b}$ modes are suppressed. But $h h \rightarrow W W^{*} b \bar{b}$ decay could provide some sensitivity, which we discuss toward the end of this section.
We set all $\rho_{i j}=0$ except $\rho_{t c}$ for simplicity throughout this section. Because of the presence of nonzero $c_{\gamma}$, the branching ratios of $h$ will be modified compared to the SM, albeit in a minor way. Since we set all $\rho_{i j}=0$ except $\rho_{t c}$ and assume $\left|c_{\gamma}\right|<0.2$, the branching ratios remain practically the same. In the following, we assume all branching ratios of $h$ are SM-like for simplicity.

To illustrate the discovery potential of $c g \rightarrow t H \rightarrow t h h$, we choose six benchmark points (BPs) from Fig. 1 with large $\left|\lambda_{H h h}\right|$ values, which are summarized in Table I. The first three, $\mathrm{BP} 1, \mathrm{BP} 2$, and $\mathrm{BP} 3$, are for the $m_{H}<m_{H^{ \pm}}, m_{A}$ scenario, while the other three, $\mathrm{BP} a, \mathrm{BP} b$, and $\mathrm{BP} c$, are for the $\left|m_{A}-m_{H}\right|<5 \mathrm{GeV}$ scenario. The values of $m_{H}$ are chosen for $2 m_{h}<m_{H}<300 \mathrm{GeV}, m_{H} \approx 300 \mathrm{GeV}$, and $m_{H}>2 m_{t}$ (above the $t \bar{t}$ threshold), respectively. All six benchmark points are for $\eta_{345}<0$, in accordance with the discussions in the preceding section to achieve large $\left|\lambda_{H h h}\right|$.

The $c g \rightarrow t H \rightarrow t h h$ process depends also on $\rho_{t c}$. For sizable $c_{\gamma}$, the available parameter space for $\rho_{t c}$ is constrained by the $\mathcal{B}(t \rightarrow c h)$ measurement. The latest ATLAS 95\% C.L. upper limit (with $13 \mathrm{TeV} 36.1 \mathrm{fb}^{-1}$ data) is $\mathcal{B}(t \rightarrow c h)<1.1 \times 10^{-3}$ [28]. Using this limit and our $c_{\gamma}$ value, we find the upper limit on $\rho_{t c}=0.54$, applicable to all six benchmark points. We find the $2 \sigma$ upper limits on $\rho_{t c}$ for $\mathrm{BP} 1, \mathrm{BP} 2$, and $\mathrm{BP} 3$ to be $0.56,0.55$, and 0.63 , respectively. The $\mathrm{BP} a, \mathrm{BP} b$, and $\mathrm{BP} c$ benchmark points were chosen such that the constraint from Ref. [25] becomes much weaker due to cancellation between 
TABLE I. Parameter values for the six benchmark points of Table I. See the text for details.

\begin{tabular}{|c|c|c|c|c|c|c|c|c|c|c|c|c|c|c|c|}
\hline $\mathrm{BP}$ & $\eta_{1}$ & $\eta_{2}$ & $\eta_{3}$ & $\eta_{4}$ & $\eta_{5}$ & $\eta_{345}$ & $\eta_{6}$ & $\eta_{7}$ & $\begin{array}{c}m_{H^{ \pm}} \\
(\mathrm{GeV})\end{array}$ & $\begin{array}{c}m_{A} \\
(\mathrm{GeV})\end{array}$ & $\begin{array}{c}m_{H} \\
(\mathrm{GeV})\end{array}$ & $c_{\gamma}$ & $s_{\gamma}$ & $\begin{array}{l}\left|\lambda_{H h h}\right| \\
(\mathrm{GeV})\end{array}$ & $\frac{\mu_{22}^{2}}{v^{2}}$ \\
\hline 1 & 0.287 & 2.996 & -0.188 & 2.039 & -2.555 & -0.704 & -0.172 & 0.557 & 303.27 & 480.96 & 279.49 & 0.169 & -0.986 & 96.80 & 1.61 \\
\hline 2 & 0.294 & 2.781 & 0.269 & 2.095 & -2.945 & -0.581 & -0.21 & 0.633 & 340.18 & 518.26 & 303.48 & 0.169 & -0.986 & 104.32 & 1.77 \\
\hline 3 & 0.309 & 2.984 & -0.017 & 2.416 & -2.727 & -0.328 & -0.301 & 0.881 & 362.90 & 536.38 & 354.15 & 0.169 & -0.986 & 123.03 & 2.18 \\
\hline$a$ & 0.286 & 2.97 & 1.508 & -2.189 & -0.02 & -0.701 & -0.169 & 0.525 & 377.31 & 276.72 & 277.71 & 0.169 & -0.986 & 96.07 & 1.59 \\
\hline$b$ & 0.294 & 2.973 & 1.42 & -1.976 & -0.037 & -0.593 & -0.211 & 0.631 & 388.90 & 304.0 & 303.92 & 0.169 & -0.986 & 105.06 & 1.78 \\
\hline$c$ & 0.309 & 2.976 & 0.294 & -0.62 & -0.045 & -0.371 & -0.301 & 0.932 & 377.17 & 353.3 & 353.89 & 0.169 & -0.986 & 124.00 & 2.2 \\
\hline
\end{tabular}

TABLE II. $H$ decay branching ratios for the benchmark points.

\begin{tabular}{lccccc}
\hline \hline $\mathrm{BP}$ & $\rho_{t c}$ & $t c$ & $h h$ & $W W$ & $Z Z$ \\
\hline 1 & 0.54 & 0.698 & 0.232 & 0.049 & 0.021 \\
2 & 0.54 & 0.688 & 0.238 & 0.051 & 0.023 \\
3 & 0.54 & 0.677 & 0.235 & 0.06 & 0.027 \\
$a$ & 0.54 & 0.700 & 0.229 & 0.049 & 0.021 \\
$b$ & 0.54 & 0.686 & 0.240 & 0.051 & 0.023 \\
$c$ & 0.54 & 0.674 & 0.238 & 0.059 & 0.027 \\
\hline \hline
\end{tabular}

$c g \rightarrow t A \rightarrow t t \bar{c}$ and $c g \rightarrow t H \rightarrow t t \bar{c} \quad[13,26]$. However, besides the aforementioned $t \rightarrow c h$ constraint, $\rho_{t c}$ can still be constrained by $B_{s, d}$ mixing and $\mathcal{B}\left(B \rightarrow X_{s} \gamma\right)$, where $\rho_{t c}$ enters via the charm loop through $H^{+}$coupling $[16,18]$. A reinterpretation of the result from Ref. [18] finds $\left|\rho_{t c}\right| \lesssim 1.7$ for $m_{H^{ \pm}}=500 \mathrm{GeV}$ [16]. In our analysis, we choose $\rho_{t c}=$ 0.54 for all six benchmark points, where the $H$ decay branching ratios are given in Table II. ${ }^{1}$

We remark that the FCNH $t u H$ coupling ${ }^{2} \rho_{t u}$ can also induce top-assisted di-Higgs via $u g \rightarrow t H \rightarrow t h h$, and our analysis can be extended to the case in which all $\rho_{i j}=0$ except $\rho_{t u}$ (see also Ref. [27]). While the ATLAS 95\% C.L. upper limit $\mathcal{B}(t \rightarrow u h)<1.2 \times 10^{-3}$ [28] is not much different from the $t \rightarrow c h$ case, the CMS four-top search [25] would give a stronger limit on $\rho_{t u}$ than the $\rho_{t c}$ case. The latter is because the relevant process $q g \rightarrow t H \rightarrow t t \bar{q}$ $(q=u, c)$ is enhanced by the parton distribution function (PDF) of the up quark, while the signal region does not differentiate $\bar{u}$ and $\bar{c}$. Similarly and more efficiently, the $t$ channel scalar exchange process $q q \rightarrow t t$ via $\rho_{t q}$ is enhanced by up-quark PDF; hence, the ATLAS same-sign top search [29] may provide a significant constraint in contrast to the $\rho_{t c}$ case [13]. Despite stronger constraints on $\rho_{t u}$, the discovery potential of $u g \rightarrow t H \rightarrow t h h$ would be balanced to some extent by the similar up-PDF enhancement in comparison to the $\rho_{t c}$ case, but, we do not expect improvement in the signal significance for BP1, BP2, and $\mathrm{BP} 3$. By contrast, for an equivalent of $\mathrm{BP} a, \mathrm{BP} b$, or $\mathrm{BP} c$,

\footnotetext{
${ }^{1}$ Note that for BP3 and BPc the coupling $\lambda_{t} c_{\gamma}$ induces $H \rightarrow t \bar{t}$ decay. However, for both of the benchmark points $\mathcal{B}(H \rightarrow t \bar{t}) \lesssim$ 0.001 and are not shown in Table II.

${ }^{2} \rho_{u t}$ is tightly constrained by $B_{d}$ mixing and $b \rightarrow d \gamma$ [18].
}

$u g \rightarrow t A \rightarrow t t \bar{u} \quad$ effectively cancels $\quad u g \rightarrow t H \rightarrow t t \bar{u}$, relaxing the four-top search limit. The same is true for the ATLAS $q q \rightarrow t t$ limit. In such a case, under the $t \rightarrow u h$ constraint, $\rho_{t u}$ may be as large as the $\rho_{t c}$ case; hence, we expect a better discovery potential of $u g \rightarrow t H \rightarrow t h h$, boosted by up the PDF.

To investigate the discovery potential of top-assisted diHiggs production at the LHC, we study $p p \rightarrow t H+X \rightarrow$ $t h h+X$ with both $h$ decaying to $b \bar{b}$, while $t \rightarrow b \ell^{+} \nu_{\ell}$. The dominant backgrounds are $t \bar{t}+$ jets, single-top, $t \bar{t} h, 4 t$, $t \bar{t} W$, and $t \bar{t} Z$, while $t Z j$, Drell-Yan + jets (DY + jets), $W+$ jets, and $t W h$ are subdominant. We do not include backgrounds from nonprompt and fake sources, as these are not properly modeled in Monte Carlo simulations and require data to estimate. We generate signal and background event samples at leading order (LO), utilizing Monte Carlo event generator MADGRAPH5_AMC@NLO [30] with default PDF set NN23LO1 [31] for $p p$ collisions at $\sqrt{s}=14 \mathrm{TeV}$, interfaced with PYTHIA6.4 [32] for showering and hadronization, and adopt the MLM matching scheme [33] for matrix element and parton shower merging. The event samples are then fed into DELPHES3.4.0 [34] for detector effects (ATLAS based). The effective Lagrangian is implemented using FEYNRULES2.0 [35].

The $t \bar{t}+$ jets background cross section is normalized to the next-to-next-to-leading-order (NNLO) ones by a factor of 1.84 [36]. The LO Wt component of the single-top cross section is normalized to next-to-leading order (NLO) by a factor of 1.35 [37], while $t$ and $s$ channels are normalized by factors of 1.2 and 1.47 , respectively [38]. The $4 t, t \bar{t} h$, $t \bar{t} W$, and $t \bar{t} Z$ cross sections at LO are adjusted to the NLO ones by factors of 2.04 [30], 1.27 [39], 1.35 [40], and 1.56 [41]. The DY + jets background is normalized to NNLO cross sections by a factor of $1.27[42,43]$. The $t W h$ and $W+$ jets background are kept at LO. The correction factors for conjugate processes are assumed to be the same for simplicity. Note that we do not include the correction factor for the LO signal cross sections.

To distinguish signal from background, we apply the event selection criteria as follows. Each event should contain one lepton and at least five jets, out of which at least four are $b$ tagged (denoted as $4 b 1 \ell$ ). This reduction in the required number of $b$ jets, from five (one from top decay and four from the $h$ decays) to four [27], is in consideration 
TABLE III. Signal and total background (bkg.) cross sections after selection cuts for the $4 b 1 \ell$ process for the benchmark points of Table I, in which the last column gives the significance for 600 (3000) $\mathrm{fb}^{-1}$ integrated luminosity.

\begin{tabular}{lccc}
\hline \hline BP & Signal $(\mathrm{fb})$ & Total bkg. $(\mathrm{fb})$ & $\begin{array}{c}\text { Significance } \\
600(3000)\left(\mathrm{fb}^{-1}\right)\end{array}$ \\
\hline 1 & 0.396 & 9.002 & $3.2(7.2)$ \\
2 & 0.38 & 9.86 & $2.9(6.6)$ \\
3 & 0.288 & 10.915 & $2.1(4.8)$ \\
$a$ & 0.39 & 8.906 & $3.2(7.1)$ \\
$b$ & 0.368 & 9.948 & $2.8(6.4)$ \\
$c$ & 0.295 & 10.898 & $2.2(4.9)$ \\
\hline \hline
\end{tabular}

of the finite $b$-tagging efficiency. The transverse momentum $\left(p_{T}\right)$ of the lepton should be $>28 \mathrm{GeV}$, while $p_{T}>$ $20 \mathrm{GeV}$ for all five jets. The pseudorapidity $(\eta)$ of the lepton and all jets should be $|\eta|<2.5$. We reconstruct jets by the anti- $k_{T}$ algorithm with radius parameter $R=0.6$. The minimum separation $(\Delta R)$ between any pair of jets, or between the lepton and any jet, should be $>0.4$. The $E_{T}^{\text {miss }}$ is required to be greater than $35 \mathrm{GeV}$.

To reduce backgrounds further, we construct all possible $m_{b b}$ combinations from the four leading $b$ jets and demand that the two $m_{b b}$ pairs that are closest to $m_{h}$ should lie within $100 \mathrm{GeV} \leq m_{b b} \leq 150 \mathrm{GeV}$. Finally, we demand the invariant mass of the four leading $b$ jets $\left(m_{4 b}\right)$ to be within $\left|m_{H}-m_{4 b}\right|<100 \mathrm{GeV}$. Note that in our exploratory study we have not optimized the $m_{4 b}$ cut for each of the benchmark points out of simplicity. We adopt the $p_{T}$ and $\eta$ dependent $b$-tagging efficiency and $c$ - and light-jet misidentification efficiencies of DELPHES. The signal and total background cross sections after selection cuts are summarized in Table III, while individual components of backgrounds are given in Table IV.

We estimate the statistical significance given in Table III by the use of $\mathcal{Z}=\sqrt{2[(S+B) \ln (1+S / B)-S]}$ [44], where $S$ and $B$ are the numbers of signal and background events after selection cuts. We find that, with $600 \mathrm{fb}^{-1}$ data, the significance can reach above approximately $3.2 \sigma$ for $\mathrm{BP} 1$ and $\mathrm{BP} a$, approximately $2.8 \sigma$ for $\mathrm{BP} 2$ and $\mathrm{BP} b$, but only approximately $2 \sigma$ for $\mathrm{BP} 3$ and $\mathrm{BP} c$. With $3000 \mathrm{fb}^{-1}$ at the HL-LHC, the significance can reach beyond $7 \sigma$ for BP1 and $\mathrm{BP} a$, about $6.5 \sigma$ for $\mathrm{BP} 2$ and $\mathrm{BP} b$, and just below $5 \sigma$ for $\mathrm{BP} 3$ and $\mathrm{BP} c$. The significance depends heavily on the choice of $\lambda_{H h h}$ and $\rho_{t c}$. To get a feeling, we rescaled the significance of the BPs by $\lambda_{H h h}=70 \mathrm{GeV}$ (denoted by red dashed line in Fig. 1) with $c_{\gamma}$ and $\rho_{t c}$ fixed as in Table I and Table II, respectively. We find approximately $4.2 \sigma$ is possible for $\mathrm{BP} 1$ and $\mathrm{BP} a$ and approximately $3.2 \sigma$ for $\mathrm{BP} 2$ and $\mathrm{BP} b$. The significance is below $2 \sigma$ for both $\mathrm{BP} 3$ and $\mathrm{BP} c$. Note that $\lambda_{H h h}=70 \mathrm{GeV}$ is possible even for lower values of $c_{\gamma}$. A lower $c_{\gamma}$ allows larger $\rho_{t c}$ for $\mathrm{BP} a$, $\mathrm{BP} b$, and BPc. Take $c_{\gamma}=0.15$ e.g., $\rho_{t c}=0.61$ (where one can achieve approximately $5 \sigma, 3.8 \sigma$, and $2.3 \sigma$, respectively, for $\mathrm{BP} a, \mathrm{BP} b$, and $\mathrm{BP} c$ with $3000 \mathrm{fb}^{-1}$ ) is allowed. Though this is not as good as those shown in Table III, it illustrates the chance for finding some signal for lower $\lambda_{H h h}$ values, but compensated by gains in $\rho_{t c}$. BP1, BP2, and BP 3 do not have this feature, as discussed earlier. In general, discovery is possible for $270 \mathrm{GeV} \lesssim m_{H} \lesssim 330 \mathrm{GeV}$ with $\lambda_{H h h}=$ $70 \mathrm{GeV}$ for $\left|m_{A}-m_{H}\right|<5 \mathrm{GeV}$, while the significance drops for the $m_{H}<m_{H^{ \pm}}, m_{A}$ scenario. As the parton luminosities falter away, the significance drops rapidly if $m_{H} \gtrsim 340 \mathrm{GeV}$ for both scenarios.

Before closing, let us mention briefly the prospect for $p p \rightarrow t H \rightarrow t h h$, where $t \rightarrow b \ell^{+} \nu_{\ell}$ but one $h$ decays to $W^{+} W^{-*}$ and the other decays to $b \bar{b}$ (conjugate process implied). Assuming the $W$ and $W^{*}$ decay leptonically, one has $3 b 3 \ell$ plus the $E_{T}^{\text {miss }}$ (denoted as $3 b 3 e$ ) signature. We find that discovery cannot be attained for any of the six benchmarks at the HL-LHC, but approximately $3.1 \sigma$ and $3 \sigma$ are possible for $\mathrm{BP} 1$ and $\mathrm{BP} 2$, reaching approximately $3 \sigma$ and $2.9 \sigma$ significance for $\mathrm{BP} a$ and $\mathrm{BP} b$, respectively. The significance for $\mathrm{BP} 3$ is $2.5 \sigma$, while it is $2.4 \sigma$ for $\mathrm{BP} c$. Here, we follow the same cut-based analysis as described in Ref. [26] for the $3 b 3 e$ process, with the additional requirement of $100 \mathrm{GeV}<m_{b b}<150 \mathrm{GeV}$. Sensitivity is poor above $m_{H} \gtrsim 320 \mathrm{GeV}$, but if one has nonzero $\rho_{t t}$, the sensitivity to $c g \rightarrow t h h$ is lost for $m_{H}>2 m_{t}$. This, however, opens up the $c g \rightarrow t H \rightarrow t t \bar{t}$ triple-top process, which also has $3 b 3 \ell$ signature but without the $100 \mathrm{GeV}<m_{b b}<$ $150 \mathrm{GeV}$ cut, which HL-LHC can actually cover [26]. Indeed, nonzero $\rho_{t t}$ motivates the conventional $g g \rightarrow H \rightarrow$ $t \bar{t}$ search or $g g \rightarrow H t \bar{t} \rightarrow t \bar{t} t \bar{t}$ [45], i.e., the four-top search. The former process suffers from large interference [46] with the overwhelming $g g \rightarrow t \bar{t}$ background; however, a recent search by ATLAS found some sensitivity [47]. It

TABLE IV. Cross sections for different background contributions after selection cuts at $\sqrt{s}=14 \mathrm{TeV}$.

\begin{tabular}{lccccccc}
\hline \hline BP & $t \bar{t}(\mathrm{fb})$ & Single top $(\mathrm{fb})$ & $t \bar{t} h(\mathrm{fb})$ & $4 t(\mathrm{fb})$ & $t \bar{t} W(\mathrm{fb})$ & $t \bar{t} Z(\mathrm{fb})$ & Others $(\mathrm{fb})$ \\
\hline 1 & 6.701 & 1.014 & 1.008 & 0.016 & 0.022 & 0.234 & 0.007 \\
2 & 7.418 & 1.014 & 1.117 & 0.019 & 0.022 & 0.262 & 0.008 \\
3 & 7.939 & 1.521 & 1.135 & 0.024 & 0.02 & 0.268 & 0.008 \\
$a$ & 6.616 & 1.014 & 1.0 & 0.016 & 0.022 & 0.231 & 0.007 \\
$b$ & 7.425 & 1.014 & 1.118 & 0.019 & 0.022 & 0.262 & 0.008 \\
$c$ & 7.923 & 1.52 & 1.135 & 0.024 & 0.02 & 0.268 & 0.008 \\
\hline \hline
\end{tabular}


should be clear, however, that $p p \rightarrow t H+X \rightarrow$ thh in $3 b 3 \ell$ can provide a supporting role in the top-assisted diHiggs program at the HL-LHC.

\section{DISCUSSION AND SUMMARY}

The 2HDM without NFC allows resonant di-Higgs production via the $c g \rightarrow t H \rightarrow t h h$ process. The process can be searched for at the LHC via $p p \rightarrow t H+X \rightarrow t h h+X$, followed by both $h$ decays to $b \bar{b}$ and $t \rightarrow b \ell^{+} \nu_{\ell}$. If all other $\rho_{i j}=0$, this process can be discovered at the HL-LHC in the mass range $270 \mathrm{GeV} \lesssim m_{H} \lesssim 360 \mathrm{GeV}$ if $\rho_{t c} \sim 0.5$ and $\lambda_{\text {Hhh }} \sim 100 \mathrm{GeV}$. The other decay modes such as $h h \rightarrow \gamma \gamma b \bar{b}, h h \rightarrow \tau \tau b \bar{b}$ are suppressed. Furthermore, $p p \rightarrow$ $t H+X \rightarrow t h h+X$ with $h h \rightarrow W^{+} W^{-*} b \bar{b}$ with $t \rightarrow b \ell^{+} \nu_{\ell}$ could be sensitive. The significances can be as large as approximately $2 \sigma-3 \sigma$ depending on the masses of $H, A$, and $H^{ \pm}$. However, both processes could be preceded by $p p \rightarrow t H+X \rightarrow t t \bar{c}$, unless $H$ and $A$ are degenerate in mass and width. In such scenarios, nonzero $\rho_{t t}$ helps via $c g \rightarrow t H \rightarrow t t \bar{t}$ [26]. In general, the presence of other Yukawas reduces the $H \rightarrow h h$ branching ratios, making discovery of top-assisted di-Higgs less likely. The cross section for $c g \rightarrow t H \rightarrow t h h$ vanishes as $c_{\gamma}$ approaches zero, and the signature requires $c_{\gamma} \sim 0.15-0.2$. If larger $\left|\eta_{i}\right|$ values are allowed beyond $3, \lambda_{H h h}$ can be enhanced even for smaller $c_{\gamma}$.

Nonzero $c_{\gamma}$ would also induce $c g \rightarrow t H \rightarrow t W^{+} W^{-}$and $c g \rightarrow t H \rightarrow t Z Z$. We find the significances of the former process lie just below $2 \sigma$ for all the benchmark points with the full HL-LHC dataset. However, for fixed values of $c_{\gamma}$ and $\rho_{t c}$, a smaller $\lambda_{H h h}$ enhances the signature for $c g \rightarrow$ $t H \rightarrow t W^{+} W^{-} \quad$ through enhanced $\mathcal{B}\left(H \rightarrow W^{+} W^{-}\right)$. Because of smaller $\mathcal{B}(Z \rightarrow \ell \ell)$, we do not find $c g \rightarrow t H \rightarrow$ $t Z Z$ to be promising for any of the benchmark points.

In summary, we have explored associated $t H \rightarrow$ thh production at the LHC via $c g \rightarrow t H \rightarrow t h h$, where production involves the extra Yukawa coupling $\rho_{t c}$, and $H \rightarrow h h$ decay needs a finite $h-H$ mixing angle $\cos \gamma \neq$ 0 as well as $\mathcal{O}(1)$ extra Higgs quartic couplings. We find a non-negligible discovery potential at HL-LHC for $m_{H} \sim 300 \mathrm{GeV}$. Considering that $h h$ production within the SM is not quite hopeful at the HL-LHC, this is an interesting result. Furthermore, a discovery might shed light on strongly first-order electroweak phase transition. If evidence is found, not only would one have discovered new physics-induced di-Higgs production, but together with the companion same-sign top signal from $c g \rightarrow t H \rightarrow t t \bar{c}$, one would be probing the $\rho_{t c}$-driven electroweak baryogenesis scenario provided by this two Higgs doublet model as well as starting to probe the associated Higgs potential.

\section{ACKNOWLEDGMENTS}

We thank K.-F. Chen and Y. Chao for fruitful discussions. This research is supported by MOST Grants No. 106-2112-M-002-015-MY3, No. 107-2811-M-002039, and No. 107-2811-M-002-3069.
[1] G. Aad et al. (ATLAS Collaboration), Phys. Lett. B 716, 1 (2012); S. Chatrchyan et al. (CMS Collaboration), Phys. Lett. B 716, 30 (2012).

[2] G. Aad et al. (ATLAS and CMS Collaborations), J. High Energy Phys. 08 (2016) 045.

[3] M. Grazzini, G. Heinrich, S. Jones, S. Kallweit, M. Kerner, J. M. Lindert, and J. Mazzitelli, J. High Energy Phys. 05 (2018) 059.

[4] LHC Higgs Cross Section HH Sub-group, https://twiki.cern .ch/twiki/bin/view/LHCPhysics/LHCHXSWGHH\#LHC_ Higgs_Cross_Section_HH_Sub_g.

[5] M. Aaboud et al. (ATLAS Collaboration), J. High Energy Phys. 01 (2019) 030.

[6] M. Aaboud et al. (ATLAS Collaboration), J. High Energy Phys. 11 (2018) 040.

[7] M. Aaboud et al. (ATLAS Collaboration), Eur. Phys. J. C 78, 1007 (2018).

[8] A. M. Sirunyan et al. (CMS Collaboration), Phys. Lett. B 778, 101 (2018).

[9] A. M. Sirunyan et al. (CMS Collaboration), J. High Energy Phys. 01 (2018) 054.
[10] A. M. Sirunyan et al. (CMS Collaboration), Phys. Lett. B 788, 7 (2019).

[11] CMS Collaboration, CERN Report No. CMS-PAS-HIG-17017, 2018.

[12] S. L. Glashow and S. Weinberg, Phys. Rev. D 15, 1958 (1977).

[13] W.-S. Hou, M. Kohda, and T. Modak, Phys. Lett. B 786, 212 (2018).

[14] K. Fuyuto, W.-S. Hou, and E. Senaha, Phys. Lett. B 776, 402 (2018).

[15] See, e.g., S. Davidson and H. E. Haber, Phys. Rev. D 72, 035004 (2005).

[16] B. Altunkaynak, W.-S. Hou, C. Kao, M. Kohda, and B. McCoy, Phys. Lett. B 751, 135 (2015).

[17] W.-S. Hou and M. Kikuchi, Eur. Phys. Lett. 123, 11001 (2018).

[18] A. Crivellin, A. Kokulu, and C. Greub, Phys. Rev. D 87, 094031 (2013).

[19] J. F. Gunion and H. E. Haber, Phys. Rev. D 67, 075019 (2003).

[20] D. Eriksson, J. Rathsman, and O. Stal, Comput. Phys. Commun. 181, 189 (2010). 
[21] M. E. Peskin and T. Takeuchi, Phys. Rev. D 46, 381 (1992).

[22] C. D. Froggatt, R. G. Moorhouse, and I. G. Knowles, Phys. Rev. D 45, 2471 (1992).

[23] H. E. Haber and O. Stål, Eur. Phys. J. C 75, 491 (2015).

[24] M. Baak and R. Kogler, arXiv:1306.0571.

[25] A. M. Sirunyan et al. (CMS Collaboration), Eur. Phys. J. C 78, 140 (2018).

[26] M. Kohda, T. Modak, and W.-S. Hou, Phys. Lett. B 776, 379 (2018).

[27] M. Buschmann, J. Kopp, J. Liu, and X.-P. Wang, J. High Energy Phys. 06 (2016) 149.

[28] M. Aaboud et al. (ATLAS Collaboration), arXiv: 1812.11568.

[29] G. Aad et al. (ATLAS Collaboration), J. High Energy Phys. 10 (2015) 150.

[30] J. Alwall, R. Frederix, S. Frixione, V. Hirschi, F. Maltoni, O. Mattelaer, H.-S. Shao, T. Stelzer, P. Torrielli, and M. Zaro, J. High Energy Phys. 07 (2014) 079.

[31] R. D. Ball, V. Bertone, S. Carrazza, L. Del Debbio, S. Forte, A. Guffanti, N. P. Hartland, and J. Rojo (NNPDF Collaboration), Nucl. Phys. B877, 290 (2013).

[32] T. Sjöstrand, S. Mrenna, and P. Skands, J. High Energy Phys. 05 (2006) 026.

[33] J. Alwall et al., Eur. Phys. J. C 53, 473 (2008).

[34] J. de Favereau, C. Delaere, P. Demin, A. Giammanco, V. Lemaître, A. Mertens, and M. Selvaggi (DELPHES 3 Collaboration), J. High Energy Phys. 02 (2014) 057.
[35] A. Alloul, N. D. Christensen, C. Degrande, C. Duhr, and B. Fuks, Comput. Phys. Commun. 185, 2250 (2014).

[36] ATLAS-CMS recommended $t \bar{t}$ cross section predictions: https://twiki.cern.ch/twiki/bin/view/LHCPhysics/ TtbarNNLO.

[37] N. Kidonakis, Phys. Rev. D 82, 054018 (2010).

[38] ATLAS-CMS recommended predictions for single-top cross sections using the HATHOR2.1 program: https://twiki.cern .ch/twiki/bin/view/LHCPhysics/SingleTopRefXsec.

[39] SM Higgs production cross sections at $\sqrt{s}=14 \mathrm{TeV}$ : https://twiki.cern.ch/twiki/bin/view/LHCPhysics/ CERNYellowReportPageAt14TeV2010.

[40] J. M. Campbell and R. K. Ellis, J. High Energy Phys. 07 (2012) 052.

[41] J. Campbell, R. K. Ellis, and R. Röntsch, Phys. Rev. D 87, 114006 (2013).

[42] Y. Li and F. Petriello, Phys. Rev. D 86, 094034 (2012).

[43] W.-S. Hou, M. Kohda, and T. Modak, Phys. Rev. D 96, 015037 (2017).

[44] G. Cowan, K. Cranmer, E. Gross, and O. Vitells, Eur. Phys. J. C 71, 1554 (2011).

[45] N. Craig, J. Hajer, Y.-Y. Li, T. Liu, and H. Zhang, J. High Energy Phys. 01 (2017) 018.

[46] For a recent reference, see M. Carena and Z. Liu, J. High Energy Phys. 11 (2016) 159, and references therein.

[47] M. Aaboud et al. (ATLAS Collaboration), Phys. Rev. Lett. 119, 191803 (2017). 